\title{
Dependability analysis of a very large volume neutrino telescope
}

\author{
Ioannis A. Papazoglou and Eftychia C. Marcoulaki* \\ System Reliability and Industrial Safety Laboratory \\ National Centre for Scientific Research "DEMOKRITOS" \\ PO Box 60228, 15310 Aghia Paraskevi, Greece
}

\section{ABSTRACT}

This work considers a first order approximation to the dependability analysis of complex large scale installations. The dependability criterion used here is quantitative unavailability, and an appropriate unavailability model is presented. The model assumes that the system is symmetrical, has various levels of hierarchy, and components found in the same level are similar and function independently.

The application example comes from very large volume neutrino telescopes installed under water or ice, consisting of several thousands of optical modules. The readout architecture of the detector has several levels of multiplexing including optical detection towers, branches and tower sectors. The paper presents results for various alternative detector layouts and distances of the detector from the onshore facilities. It also develops dependability requirements for major components and/or subsystems consistent with an overall system performance target. The results depict the dependence of the system unavailability on the number of optical modules and the alternative deep sea infrastructure configurations for transferring the measured signals.

Keywords: large neutrino telescope, reliability, availability allocation

* Corresponding author. Tel: +302106503743; fax: +302106548415.

E-mail addresses: yannisp@ipta.demokritos.gr (Ioannis A. Papazoglou), emarcoulaki@ipta.demokritos.gr (Eftychia C. Marcoulaki). 


\section{INTRODUCTION}

Astronomical objects can be observed using Cherenkov telescopes to detect the highenergy astrophysical neutrinos they emit. In Cherenkov telescopes water or ice has the dual role of absorbing daylight and the vast majority of charged cosmic rays, while allowing the detection of atmospheric or astrophysical neutrinos through the Cherenkov light emitted by muonic reaction products in aqueous transparent media ${ }^{[1]}$. These telescopes employ a grid of several thousands of Optical Modules (OM) to measure this light and enable optical neutrino detection. According to solid theoretical arguments, the total volume of the detector grid should be at least of the scale of a cubic kilometer, as in the cases of IceCube ${ }^{[2]}$ which is expected to be complete in 2011 and $\mathrm{KM} 3 \mathrm{NeT}^{[3]}$ which is in preparatory phase ${ }^{[4]}$. The OMs consist of the photon sensors and the associated electronics housed in a glass sphere. To form a spatial grid, the OMs are placed on mechanical structures, the Detection Units (DUs), supporting and connecting them in vertical assemblies. The OMs are interconnected via watertight and pressure-resistant connectors, and their measured data travel through an underwater network of multiplexed passive optical or active electronic equipment and optical fibers to the onshore base ${ }^{[5]}$.

Dependability is the ability of a system to perform its required function, under determined operating conditions, during a given period of operation. The dependability measure used here is the overall telescope unavailability, namely the percentage of time that the telescope is expected to be unavailable given the desired period of operation. The telescope is treated as a complex system; therefore its unavailability can be expressed as a function of the unavailabilities of the telescope components. The following sections discuss the development of an appropriate model to determine: (a) the system unavailability given the unavailabilities of its components, and (b) the component unavailabilities that satisfy a given performance criterion.

The paper is organized as follows. Section 2 describes the main relevant characteristics of the neutrino telescope. Section 3 presents the method of analysis and the model linking the component unavailabilities with the system unavailability. Section 4 presents and discusses some numerical results, and section 5 concludes this work. 


\section{SYSTEM DESCRIPTION}

The large volume neutrino telescope consists of various components and subsystems that can be distinguished into three major subsystems: the detector network, the deep sea infrastructure and the onshore infrastructure ${ }^{[5]}$ (see Figure 1). All three subsystems must be successful for the telescope to be successful. This work considers the dependability analysis of the OM network and the deep-sea infrastructure. Depending on the specific design, the components belonging to each of the above subsystems and their interconnection layouts may differ. This section provides a conceptual description of the telescope's detector grid and cabling.

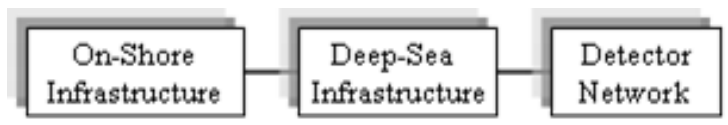

Figure 1: Overall neutrino telescope system

The Detector Network consists of the OMs grouped to form DUs, the DUs grouped into branches, and branches into sectors, as described below. The OM block contains a multiple photomultiplier tube (PMT), the associated electronics and all the cabling connecting it with the Junction Box (JB) of the DU. At the onshore base the data will be processed, stored and analyzed further to find hit patterns corresponding to neutrino-generated muon tracks producing light in the water ${ }^{[8]}$.

The readout architecture of the detector has several levels of multiplexing, starting from the glass sphere connector where the analogue PMT electrical signal is digitized. At each DU, the digitized outputs of the optical modules are gathered in the JB of the DU. This JB contains all the necessary electronics to process the signals from the OMs and the necessary cabling for the signals to travel to shore. Operability of a DU is not defined in terms of the number of the available OM since, as mentioned previously, only the total number of available OMs in the system is of importance. A DU is unavailable if there is a structural failure and the DU collapses, or when the JB of the DU fails, or when the connection of the JB to the DU sector cable fails. The output signals of the DU JBs travel through a cable network to the Primary Junction Box(es) (PJB(s)). Apart from optical fibers, all the network cables include electrical wires, to provide the power supply necessary for the operation of the telescope. The DUs are placed on the seabed forming a rectangular layout. Figure 2 gives a 3D ground plan view of the rectangular arrangement of the DU grid on the seabed assuming a set of two PJBs operating in parallel. On each horizontal string of the grid, the DUs are connected on the same 
cable, starting from the PJB1 and finishing in PJB2. The cable contains optical fibers and each fiber can serve more than one DU, as Figure 2 illustrates. DUs are grouped into sectors according to the cable they are connected to, and sector branches according to the cable fiber they are connected to. A branch is unavailable when the fiber connecting the DUs is unavailable. Likewise, a sector is unavailable when the sector cable connecting the two primary junction boxes is unavailable. The Deep-Sea Infrastructure consists of the PJB(s) and one main cable carrying the output signal of the PJB(s) to the onshore base.

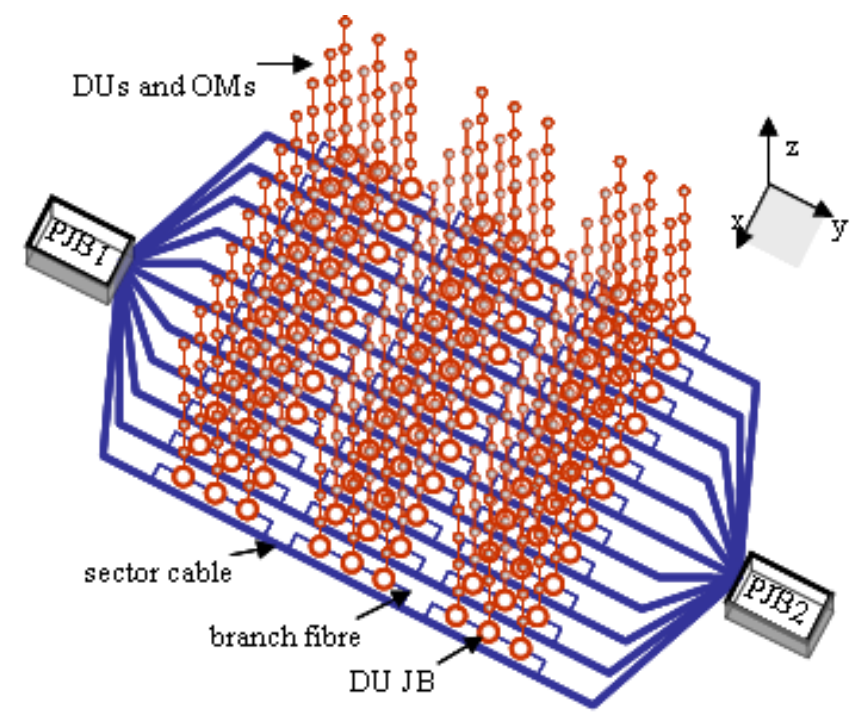

Figure 2: Three-dimensional detector network layout

In dependability analysis it is essential to determine the required function of the system under consideration. The objective of the neutrino telescope is to record and analyze neutrino interactions with matter. Simulation experiments with alternative geometries of the conceptual design have established that the telescope performance depends mainly on the number of available OMs and not on their spatial distribution (within a given number of available geometries) ${ }^{[5-7]}$. For this reason it is reasonable to assume here that: the required function of the telescope is to have an appropriate number of OMs operable and available to detect neutrino interactions and to transfer their measured data back to shore.

\section{UNAVAILABILITY MODEL}

The development of the DN unavailability model is based on (a) the network component failure assumptions discussed in the previous section, (b) a success criterion based on the minimum number of available OMs and (c) a set of design assumptions described bellow. It is 
also assumed that all the system components can be in one of the two states: available or unavailable to perform their required operations.

The functional relationship of the various parts of the DN is developed as a hierarchical structure made up of four layers of components: the top layer is the sector cables, then the sector cable fibers (or branches) and the DUs, and finally the OMs as the lowest level layer. Failure of a top layer component renders unavailable all the lower level components associated with the failed component. Therefore, when a sector component fails all the branches, DUs and OMs on the sector become unavailable. Similarly, all the DUs and OMs of a failed branch are unavailable, and all the OMs of a failed DU are unavailable. However, component failures do not affect other components on the same or higher layers. Another important assumption is that all the same layer components are identical, meaning that they have the same reliability, maintenance and repair features, and that they are connected to the same number of lower layer(s) components. Therefore, all the sectors, branches and DUs are connected to the same number of branches, DUs and OMs, respectively. Note that, all the assumptions are in good agreement with the generic features of the KMeNeT telescope as it currently being designed ${ }^{[7]}$.

The overall logical interconnection of the system components is given in Figure 3.

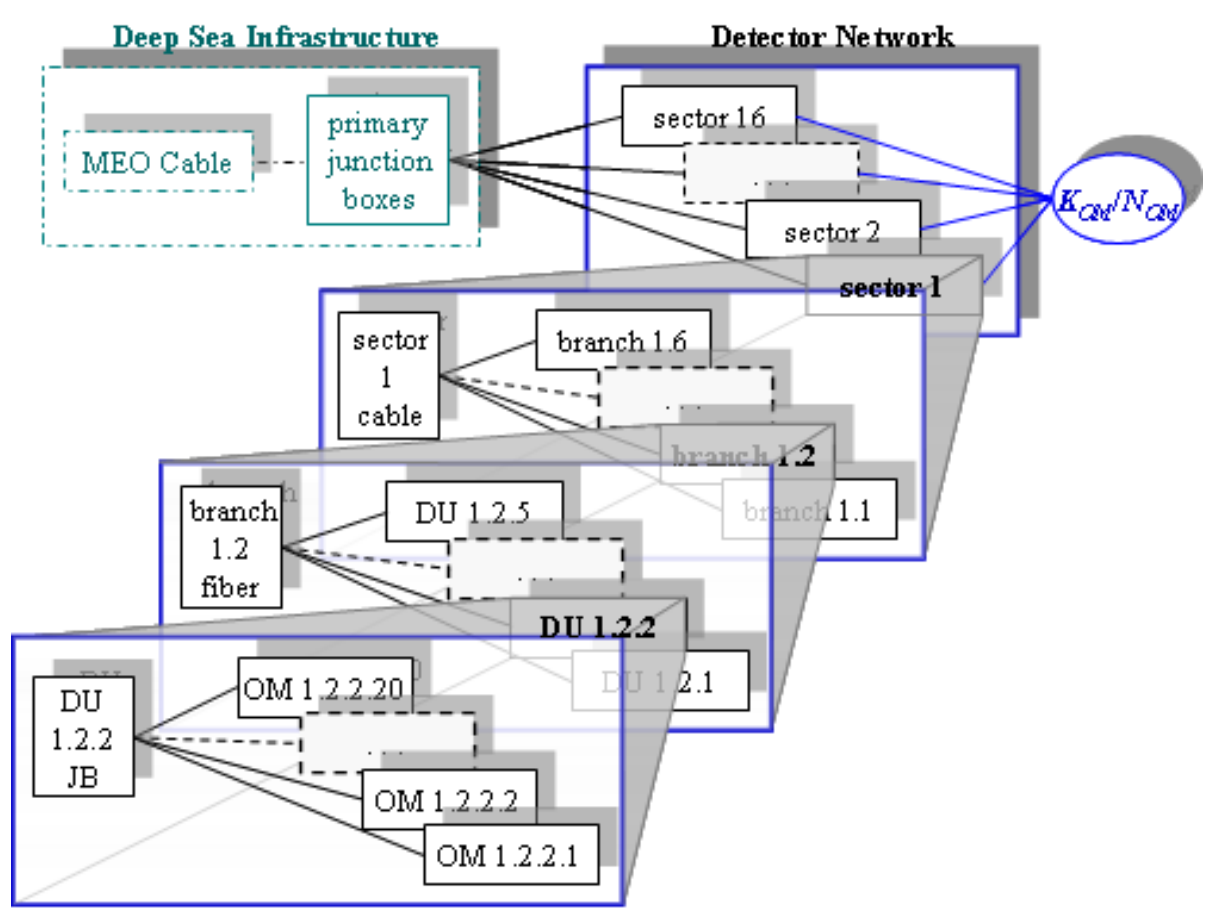

Figure 3: Detector network block diagrams 


\subsection{General Considerations}

The unavailability of the DN has been calculated as a function of the unavailability of its components under the following assumption: The unavailability of each and every component is assumed constant and equal to the average unavailability over the period of analysis (lifetime of the telescope). As a result of this assumption, the unavailability of the system is also constant and it can be calculated through static reliability analysis. This a first approximation of the systems operating behavior and it aims at identifying a first approximation of the order of magnitude of the various dependability characteristics of the components. These in turn, will be set as design and manufacturing targets for the various components of the system. Depending on the feasibility of achieving these dependability goals by the various components, the final design (i.e. number of OMs and overall size of the telescope) will be determined.

Recursive formulae are developed to express the unavailability of the system in terms of the unavailability of the components in each layer. In this part of the analysis no assumption is made about the nature of the failure and/or repair rates of the components, or the testing and repair methods. In the remaining of this subsection, a number of general relationships used in the unavailability derivation are presented.

Let $N_{k}$ be the number of components of type $k$ in the DN, $k \in\{1,2,3,4\}$, where indices 1,2 , 3 and 4 denote sectors, branches, DUs and OMs, respectively. Let $n_{k}$ be the number of components of layer $k$ connected to each component of layer $k-1$, and $n_{1}=N_{1}$. Then

$$
N_{k}=N_{d} \cdot \prod_{i=d+1}^{k} n_{i}, \forall d \leq k
$$

Let $K_{O M}$ denote the minimum number of available OMs for the telescope system to be available. Then the maximum percentage, $c$, of OMs that if failed the system is still considered available is a known quantity given as $c \equiv\left(N_{4}-K_{O M}\right) / N_{4}$.

The development of the DN model follows the system hierarchy described above, starting from the top layer and proceeding to the lower layers. Let $m_{k}$ denote the number of failed layer $k$ components, and $m_{i}^{*}$ be the maximum $m_{k}$ for the system to be available.

Since failures of same layer components occur independently, the probability of having a certain number of failed components on layer $k$ is calculated as follows. Consider the set of the mutually exclusive events 


$$
X_{k}=0, X_{k}=1, \ldots, X_{k}=m_{k}, \text { and } X_{k}>m_{k}
$$

The probability of up to and including $m_{k}$ components being unavailable is given by ${ }^{\text {[9] }}$

$$
\operatorname{Pr}\left[X_{k} \leq m_{k}\right]=\sum_{i_{k}=0}^{m_{k}} \operatorname{Pr}\left[X_{k}=i_{k}\right]
$$

Assuming that the events $X_{k}$ follow the binomial probability distribution, for a sample of $N_{k}$ identical components with probability $p_{k}$ for each component to be unavailable

$$
P\left[X_{k}=i_{k}\right]=\left(\begin{array}{c}
N_{k} \\
i_{k}
\end{array}\right) p_{k}{ }^{i} \cdot\left(1-p_{k}\right)^{N_{k}-i_{k}}
$$

and equation (2) becomes

$$
\operatorname{Pr}\left[X_{k} \leq m_{k}\right]=\sum_{i_{k}=0}^{m_{k}}\left(\begin{array}{c}
N_{k} \\
i_{k}
\end{array}\right) p_{k}^{i_{k}} \cdot\left(1-p_{k}\right)^{N_{k}-i_{k}}
$$

The probability of over $m_{k}$ components being unavailable is

$$
\operatorname{Pr}\left[X_{k}>m_{k}\right]=1-\sum_{i_{k}=0}^{m_{k}}\left(\begin{array}{c}
N_{k} \\
i_{k}
\end{array}\right) p_{k}{ }^{i_{k}} \cdot\left(1-p_{k}\right)^{N_{k}-i_{k}}
$$

The following subsections describe the development of the detector model starting from the top layer components (sectors), down to the lowest layer (OMs).

\subsection{Layer 1: Sector component}

Let $X_{1}$ denote the number of unavailable sectors and $m_{1}^{*}$ be the maximum number of unavailable sectors for the system to be operational (see Figure 3). Then

$$
m_{1}^{*}=\operatorname{int}\left[c \cdot N_{1}\right]=\operatorname{int}\left[c \cdot n_{1}\right]
$$

Let $D N$ denote the event "Detector Network unavailable" and $U_{D N}$ denote the probability of event $D N$. For $m_{1}=m_{1}{ }^{*}$, consider the complete set of the mutually exclusive events

$$
X_{1}=0, X_{1}=1, \ldots, X_{1}=m_{1}, \text { and } X_{1}>m_{1}
$$

Then the $U_{D N}$ can be written as

$$
U_{D N}=\sum_{i_{1}=0}^{m_{1}}\left(\operatorname{Pr}\left[X_{1}=i_{1}, D N\right]\right)+\operatorname{Pr}\left[X_{1}>m_{1}, D N\right]
$$

where, $\operatorname{Pr}\left[X_{1}=i_{1}, D N\right]$ denotes the probability of the joint event that the system is unavailable and $i_{1}$ sectors are unavailable, while $\operatorname{Pr}\left[X_{1}>m_{1}, D N\right]$ denotes the probability of the joint event that the system is unavailable and more than $m_{1}$ sectors are unavailable. 
Expansion of the joint event probabilities in Equation (6) yields

$$
U_{D N}=\sum_{i_{1}=0}^{m_{1}} \operatorname{Pr}\left[X_{1}=i_{1}\right] \cdot U\left(D N \mid X_{1}=i_{1}\right)+\operatorname{Pr}\left[X_{1}>m_{1}\right] \cdot U\left(D N \mid X_{1}>m_{1}\right)
$$

where $U\left(D N \mid X_{1}=i_{1}\right)$ is the unavailability of the system given that $i_{1}$ sectors are unavailable, and $U\left(D N \mid X_{1}>m_{1}\right)$ is the unavailability of the system given that more than $m_{1}$ sectors are unavailable.

If more than $m_{1}$ sectors are unavailable, then the criterion for at least $K_{O M}$ available OMs is violated and the DN is unavailable, hence $U\left(D N \mid X_{1}>m_{1}\right)=1$ and equation (7) becomes

$$
U_{D N}=\sum_{i_{1}=0}^{m_{1}}\left(\operatorname{Pr}\left[X_{1}=i_{1}\right] \cdot U\left(D N \mid X_{1}=i_{1}\right)\right)+\operatorname{Pr}\left[X_{1}>m_{1}\right]
$$

Assuming that $X_{1}$ follows the binomial probability distribution, for a sample of $n_{1}$ sectors and probability $p_{1}$ for each sector to be unavailable

$$
\operatorname{Pr}\left[X_{1}=i_{1}\right]=\left(\begin{array}{c}
n_{1} \\
i_{1}
\end{array}\right) \cdot p_{1}^{i_{1}} \cdot\left(1-p_{1}\right)^{n_{1}-i_{1}}
$$

and

$$
\operatorname{Pr}\left[X_{1}>m_{1}\right]=1-\sum_{i_{1}=0}^{m_{1}} \operatorname{Pr}\left[X_{1}=i_{1}\right]
$$

\subsection{Layer 2: Branch component}

Let $m_{2}^{*}$ be the maximum number of unavailable branches with which the system is considered operating (see Figure 3). Based on equation (1)

$$
m_{2}^{*}=\operatorname{int}\left[c \cdot N_{2}\right]=\operatorname{int}\left[c \cdot n_{1} \cdot n_{2}\right]
$$

However, when $i_{1}$ sectors are unavailable $i_{1} \cdot n_{2}$ branches are also unavailable. The maximum number of branches that can be unavailable without violating the success criterion is, therefore, reduced to

$$
m_{2}\left(i_{1}\right)=m_{2}^{*}-i_{1} \cdot n_{2}
$$

Consider the complete set of mutually exclusive events

$$
X_{2}=0, \quad X_{2}=1, \ldots X_{2}=m_{2}\left(i_{1}\right), \quad \text { and } \quad X_{2}>m_{2}\left(i_{1}\right)
$$

Then the term $U\left(D N \mid X_{1}=i_{1}\right)$ of equation (8) can then be written as 


$$
\begin{aligned}
U\left(D N \mid X_{1}=i_{1}\right)= & \sum_{i_{2}=0}^{m_{2}\left(i_{1}\right)}\left(\operatorname{Pr}\left[X_{2}=i_{2} \mid X_{1}=i_{1}\right] \cdot U\left(D N \mid X_{1}=i_{1}, X_{2}=i_{2}\right)\right)+ \\
& +\operatorname{Pr}\left[X_{2}>m_{2}\left(i_{1}\right) \mid X_{1}=i_{1}\right] \cdot U\left(D N \mid X_{1}=i_{1}, X_{2}>m_{2}\left(i_{1}\right)\right)
\end{aligned}
$$

where $i_{2}$ denotes the number of unavailable branches.

Let the number of the remaining branches when $i_{1}$ sectors are down be denoted by $n_{2}^{*}\left(i_{1}\right)$ and given by

$$
n_{2}^{*}\left(i_{1}\right)=\left(n_{1}-i_{1}\right) \cdot n_{2}
$$

If more than $m_{2}\left(i_{1}\right)$ branches become unavailable, the criterion for at least $K_{O M}$ available OMs is violated and the $\mathrm{DN}$ is unavailable, thus $U\left(D N \mid X_{1}=i_{1}, X_{2}>n_{2}\right)=1$ and equation (14) writes

$$
U\left(D N \mid i_{1}\right)=\sum_{i_{2}=0}^{m_{2}(i)} \operatorname{Pr}\left[X_{2}=i_{2} \mid i_{1}\right] \cdot U\left(D N \mid i_{1}, i_{2}\right)+\operatorname{Pr}\left[X_{2}>m_{2}\left(i_{1}\right) \mid i_{1}\right]
$$

where the events $\left\{X_{\mathrm{k}}=i_{\mathrm{k}}\right\}$ have been simplified to $\left\{i_{\mathrm{k}}\right\}$.

Assuming that $X_{2}$ follows the binomial distribution, for a sample of $n_{2}^{*}\left(i_{1}\right)$ branches and probability $p_{2}$ for each branch to be unavailable

$$
\operatorname{Pr}\left[X_{2}=i_{2} \mid X_{1}=i_{1}\right]=\left(\begin{array}{c}
n_{2}^{*}\left(i_{1}\right) \\
i_{2}
\end{array}\right) \cdot p_{2}^{i_{2}} \cdot\left(1-p_{2}\right)^{n_{2}^{*}\left(i_{1}\right)-i_{2}}
$$

and

$$
\operatorname{Pr}\left[X_{2}>m_{2}\left(i_{1}\right) \mid X_{1}=i_{1}\right]=1-\sum_{i_{2}=0}^{m_{2}\left(i_{1}\right)} \operatorname{Pr}\left[X_{2}=i_{2} \mid X_{1}=i_{1}\right]
$$

\subsection{Layer 3: Detection unit component}

Let now $m_{3}^{*}$ denote the maximum number of unavailable DUs with which the system is considered operational (see Figure 3). Based on equation (1)

$$
m_{3}^{*}=\operatorname{int}\left[c \cdot N_{3}\right]=\operatorname{int}\left[c \cdot n_{1} \cdot n_{2} \cdot n_{3}\right]
$$

When $i_{1}$ sectors are unavailable and $i_{2}$ additional branches (beyond those belonging to the $i_{1}$ sectors) are unavailable, the maximum number of extra DUs that can be unavailable is

$$
m_{3}\left(i_{1}, i_{2}\right)=m_{3}^{*}-\left(i_{1} \cdot n_{2}+i_{2}\right) \cdot n_{3}
$$

Consider the complete set of mutually exclusive events

$$
X_{3}=0, X_{3}=1, \ldots, X_{3}=m_{3}\left(i_{1}, i_{2}\right), \text { and } X_{3}>m_{3}\left(i_{1}, i_{2}\right)
$$


Then the term $U\left(D N \mid i_{1}, i_{2}\right)$ of equation (16) can be written as

$$
\begin{aligned}
U\left(D N \mid i_{1}, i_{2}\right)= & \sum_{i_{3}=0}^{m_{2}\left(i_{1}, i_{2}\right)}\left(\operatorname{Pr}\left[X_{3}=i_{3} \mid i_{1}, i_{2}\right] \cdot U\left(D N \mid i_{1}, i_{2}, i_{3}\right)\right)+ \\
& +\operatorname{Pr}\left[X_{3}>m_{3}\left(i_{1}, i_{2}\right) \mid i_{1}, i_{2}\right] \cdot U\left(D N \mid i_{1}, i_{2}, X_{3}>m_{3}\left(i_{1}, i_{2}\right)\right)
\end{aligned}
$$

where $i_{3}$ denotes the number of unavailable DUs.

The number of DUs outside those in the failed $i_{1}$ sectors and $i_{2}$ branches is

$$
n_{3}^{*}\left(i_{1}, i_{2}\right)=\left(n_{2}^{*}\left(i_{1}\right)-i_{2}\right) \cdot n_{3}
$$

If more than $m_{3}\left(i_{1}, i_{2}\right)$ DUs become unavailable, the criterion for at least $K_{O M}$ available OMs is violated and the DN is unavailable, thus $U\left(D N \mid i_{1}, i_{2}, X_{3}>n_{3}\left(i_{1}, i_{2}\right)\right)=1$ and equation (21) becomes

$$
\begin{aligned}
U\left(D N \mid i_{1}, i_{2}\right) & =\sum_{i_{3}=0}^{n_{3}^{*}\left(i_{1}, i_{2}\right)}\left(\operatorname{Pr}\left[X_{3}=i_{3} \mid i_{1}, i_{2}\right] \cdot U\left(D N \mid i_{1}, i_{2}, i_{3}\right)\right)+ \\
& +\operatorname{Pr}\left[X_{3}>m_{3}\left(i_{1}, i_{2}\right) \mid i_{1}, i_{2}\right]
\end{aligned}
$$

Assuming that $X_{3}$ follows the binomial probability distribution, for a sample of $n_{3}^{*}\left(i_{1}, i_{2}\right)$ DUs and probability $p_{3}$ for each DU to be unavailable

$$
\operatorname{Pr}\left[X_{3}=i_{3} \mid i_{1}, i_{2}\right]=\left(\begin{array}{c}
n_{3}^{*}\left(i_{1}, i_{2}\right) \\
i_{3}
\end{array}\right) \cdot p_{3}^{i_{3}} \cdot\left(1-p_{3}\right)^{n_{3}^{*}\left(i_{1}, i_{2}\right)-i_{3}}
$$

and

$$
\operatorname{Pr}\left[X_{3}>m_{3}\left(i_{1}, i_{2}\right) \mid i_{1}, i_{2}\right]=1-\sum_{i_{3}=0}^{m_{3}\left(i_{1}, i_{2}\right)} \mathrm{P}\left[X_{3}=i_{3} \mid i_{1}, i_{2}\right]
$$

\subsection{Layer 4: Optical module component}

Let now $m_{4}^{*}$ denote the maximum number of unavailable OMs with which the system is considered operational (see Figure 3). Based on equation (1)

$$
m_{4}^{*}=\operatorname{int}\left[c \cdot N_{4}\right]=\operatorname{int}\left[c \cdot n_{1} \cdot n_{2} \cdot n_{3} \cdot n_{4}\right]
$$

When $i_{1}$ sectors, $i_{2}$ branches and $i_{3}$ DUs are unavailable, the maximum number of extra OMs that can be unavailable for the DN to be available is reduced to

$$
m_{4}\left(i_{1}, i_{2}, i_{3}\right)=m_{4}^{*}-\left[\left(i_{1} \cdot n_{2}+i_{2}\right) \cdot n_{3}+i_{3}\right] \cdot n_{4}
$$

Consider the complete set of the mutually exclusive events

$$
X_{4}=0, X_{4}=1, X_{4}=2, \ldots, X_{4}=m_{4}\left(i_{1}, i_{2}, i_{3}\right), \text { and } X_{4}>m_{4}\left(i_{1}, i_{2}, i_{3}\right)
$$


Then the term $U\left(D N \mid i_{1}, i_{2}, i_{3}\right)$ of equation (23) can be written as

$$
\begin{aligned}
U\left(D N \mid i_{1}, i_{2}, i_{3}\right) & =\sum_{i_{4}=0}^{m_{4}\left(i_{1}, i_{2}, i_{3}\right)}\left(\operatorname{Pr}\left[X_{4}=i_{4} \mid i_{1}, i_{2}, i_{3}\right] \cdot U\left(D N \mid i_{1}, i_{2}, i_{3}, i_{4}\right)\right)+ \\
& +\operatorname{Pr}\left[X_{4}>m_{4}\left(i_{1}, i_{2}, i_{3}\right)\right] \cdot U\left(D N \mid i_{1}, i_{2}, i_{3}, X_{4}>m_{4}\left(i_{1}, i_{2}, i_{3}\right)\right)
\end{aligned}
$$

where $i_{4}$ denotes the number of unavailable OMs.

If more than $m_{4}\left(i_{1}, i_{2}, i_{3}\right)$ OMs become unavailable, the criterion for at least $K_{O M}$ available OMs is violated and the DN is unavailable, thus $U\left(D N \mid i_{1}, i_{2}, i_{3}, X_{4}>n_{4}\left(i_{1}, i_{2}, i_{3}\right)\right)=1$. Additionally, $m_{4}^{*}$ is known and equal to $\left(N_{4}-K_{O M}\right)$, therefore, the $\mathrm{DN}$ is always available for any $i_{4} \leq m_{4}\left(i_{1}, i_{2}, i_{3}\right)$, thus $U\left(D N \mid i_{1}, i_{2}, i_{3}, i_{4}\right)=0$. Finally, equation (28) becomes

$$
U\left(D N \mid i_{1}, i_{2}, i_{3}\right)=\operatorname{Pr}\left[X_{4}>n_{4}\left(i_{1}, i_{2}, i_{3}\right)\right]
$$

The total number of remaining OMs, outside the $i_{1}$ unavailable sectors, the $i_{2}$ unavailable branches and the $i_{3}$ unavailable DUs is

$$
n_{4}^{*}\left(i_{1}, i_{2}, i_{3}\right)=\left(n_{3}^{*}\left(i_{1}, i_{2}\right)-i_{3}\right) \cdot n_{4}
$$

Using equation (30), equation (29) becomes

$$
U\left(D N \mid i_{1}, i_{2}, i_{3}\right)=1-\sum_{i_{4}=0}^{n_{4}^{*}\left(i_{1}, i_{2}, i_{3}\right)} \operatorname{Pr}\left[X_{4}=i_{4} \mid i_{1}, i_{2}, i_{3}\right]
$$

Assuming that $X_{4}$ follows the binomial probability distribution, for a sample of $n_{4}^{*}\left(i_{1}, i_{2}, i_{3}\right)$ OMs and probability $p_{4}$ for each OM to be unavailable

$$
\operatorname{Pr}\left[X_{4}=i_{4} \mid i_{1}, i_{2}, i_{3}\right]=\left(\begin{array}{c}
n_{4}^{*}\left(i_{1}, i_{2}, i_{3}\right) \\
i_{4}
\end{array}\right) \cdot p_{4}^{i_{4}} \cdot\left(1-p_{4}\right)^{n_{4}^{*}\left(i_{1}, i_{2}, i_{3}\right)-i_{4}}
$$

\subsection{System unavailability}

It follows that the DN unavailability can be calculated recursively from equations (31), (23), (16) and (8), assuming that the unavailabilities of each system component are known. Note that, the model developed here for the DN can easily be extended to systems of more than 4 layers.

Once $U_{D N}$ is determined, the overall unavailability $U_{T}$ of the serial system composed of the OM network and the deep sea infrastructure is evaluated as

$$
U_{T}=U_{D N}+U_{0}-U_{D N} \cdot U_{0}
$$

where $U_{0}$ is the combined unavailability of the $\mathrm{PJB}(\mathrm{s})$ and the cable to shore. Since these 
are connected in series, $U_{0}$ is given by

$$
U_{0}=p_{C}+p_{0}-p_{C} \cdot p_{0}
$$

where, $p_{0}$ and $p_{c}$ denote the unavailabilities of the $\operatorname{PJB}(\mathrm{s})$ and the main cable components, respectively.

\section{COMPONENT AVAILABILITY ALLOCATION}

The objective of the dependability analysis presented in this paper is to obtain a first order approximation of the required component availability characteristics in order to achieve an overall system availability requirement.

The base case design considered here is a cuboid grid of $N_{4}=17 \times 18 \times 20=6120$ OMs. The OMs are arranged in $N_{3}=306$ DUs each containing 20 OMs. The DUs are grouped into $N_{2}=$ 102 branches each containing 3 DUs. The branches are grouped into $N_{1}=17$ sectors each containing 6 branches. This configuration has $n_{1}=17, n_{2}=6, n_{3}=3$ and $n_{4}=20$ and represented by the vector $[17,6,3,20]$.

Since the performance for the telescope depends on the number of available OM regardless of their spatial distribution ${ }^{[5-7]}$, the OMs can be treated as completely identical and equivalent components regardless of their position in the telescope, and the analysis of section 3 becomes applicable. For the basic design of the OMs and the general configuration of the neutrino telescope, the following availability criteria have been established

- Mission time of the system, $T$, is 10 years.

- The minimum number of available OMs for the telescope to operate successfully is set at $90 \%$ of the OMs in the base design, thus $K_{O M}=5508$ available OMs.

- For at least $90 \%$ of the mission time more than $K_{O M}$ OMs must be available to detect neutrino interactions and transmit the corresponding information to the shore, namely $U_{T} \leq 10^{-1}$.

\subsection{Component maintenance}

One of the telescope design issues is the layout of the OMs into different configurations, in order to achieve minimum cost and maximum availability. Additional issues include the required reliability characteristics of the various components, so that the overall system dependability performance requirement is met. This section further explores these issues under the assumption that components are characterized by constant failure and repair rates. 
Two factors determine the component availability

a) the component's mean-time-to-failure (MTTF) and

b) whether the component is repairable and if yes its mean-time-to-repair (MTTR)

The same average unavailability can be achieved by a non-repairable component with long MTTF or by a repairable component with a shorter MTTF and an appropriate MTTR. An important decision in the design of the telescope is, therefore, whether the layout would be such to allow the retrievability of the various components and subsystems. At this stage of the telescope's development it appears that the OMs and the DUs won't be retrievable, for a number of technical and cost-related reasons.

In the system unavailability calculations the relationships developed in section 3 are used. Average, constant unavailability values for each component are assumed in the place of $p_{1}$ to $p_{4}$ in equations (8) to (31). This is an acceptable approximation, provided that the average or the steady-state component unavailabilities are obtained in relatively short times with respect to the system's mission time. Let $\lambda=1 / M T T F$ denote the component failure rate.

For non-repairable components operating over a period of time $T$, the average unavailability relationship is according to Rausand and Hoyland ${ }^{[10]}$

$$
\bar{p}_{a}=1-\frac{1}{\lambda T} \cdot\left(1-e^{\lambda T}\right)
$$

For repairable components where the failure is detected immediately and the repair starts also immediately with $d=M T T R$, the steady state unavailability is given by Kumamoto and Henley ${ }^{[11]}$ as

$$
\bar{p}_{b}=\frac{d}{M T T F+d}
$$

For repairable components, testable or reachable every $T_{o}$ units of time and with constant repair duration $d$, the average unavailability is given by

$$
\bar{p}_{c, \text { int }}(\infty)=1-\frac{1-e^{-\lambda\left(T_{o}+d\right)}}{\lambda\left(T_{o}+d\right)\left(1+e^{-\lambda T_{o}}-e^{-\lambda\left(T_{o}+d\right)}\right)}
$$

Proof of equation (37) is provided in the Appendix.

\subsection{Unavailability results}

This section examines a variety of OM network layouts and evaluates feasible sets of component unavailabilities for the telescope to achieve a given unavailability target. As mentioned above, the overall maximum telescope unavailability $U_{T}$ used here is set at $10^{-1}$.

In each unavailability estimation, given are the total number of optical modules and their 
network arrangement into DUs, branches, and sectors. Seven configurations are considered, all having the same DU structure $\left(n_{4}=20\right)$ and type of sector cables $\left(n_{2}=3\right)$. These are the $[17,6,3,20],[18,6,3,20],[19,6,3,20],[20,6,3,20],[19,7,3,20],[20,7,3,20]$ and $[21,7,3,20]$, corresponding to 6120 to $8820 \mathrm{OMs}$.

The following assumptions are made

- The unavailability of the main cable $p_{c}$ ranges from $10^{-3}$ to $8 \times 10^{-2}$. If the cable is reachable and immediately repairable with one month MTTR, these unavailabilities translate to MTTFs from 80 to 1 years, respectively, according to equation (36).

- The unavailability of the $\operatorname{PJB}(\mathrm{s}) p_{0}$ is fixed at $2 \times 10^{-2}$. If the $\mathrm{PJB}(\mathrm{s})$ is(are) reachable once a year and repaired for one month, the corresponding MTTF is 30 years, according to equation (37).

- The unavailability of the sector $p_{1}$ ranges from $5 \times 10^{-3}$ to $5 \times 10^{-2}$. The corresponding MTTFs for a non-repairable component featuring these unavailabilities are 1000 to 100 years, respectively, according to equation (35).

- The unavailability of the branch is always $p_{2}=5 \cdot p_{1}$, meaning that an optical fiber is assumed five times more likely to fail than the cable containing it. Their unavailability ranges form $2.5 \times 10^{-2}$ to $2.5 \times 10^{-1}$, giving non-repairable component MTTFs from 200 to 16.5 years, respectively, according to equation (35).

- The unavailability of the detection unit $p_{3}$ ranges from $7 \times 10^{-3}$ to $3 \times 10^{-1}$, yielding MTTFs for non-repairable components from 700 to 13 years, respectively, according to equation (35).

- The unavailability of the optical module $p_{4}$ ranges from $7 \times 10^{-4}$ to $3 \times 10^{-1}$, yielding MTTFs for non-repairable components from 7000 to 13 years, respectively, according to equation (35).

Since $U_{T}$ is fixed, the model of section 3 is solved to provide combinations of unavailabilities of the main cable, the $\mathrm{PJB}(\mathrm{s})$, the sectors, the branches, the DUs and the OMs, which satisfy this system unavailability value. Figures 4 to 8 present indicative results for each network layout.

Figure 4 presents the results for the $[17,6,3,20]$ configuration. For this configuration results within the assumed unavailability ranges exist only if the unavailability of the sector $\left(p_{1}\right)$ is $5 \times 10^{-3}$ or less. For non-repairable components this implies a MTTF $\geq 1000$ years. If the $p_{1}=5 \times 10^{-3}$ unavailability is achievable then the maximum main cable unavailability $\left(p_{c}\right)$ is $6 \times 10^{-2}$ and the corresponding DU and OM unavailabilities are $p_{3}=7.5 \times 10^{-3}$ and 


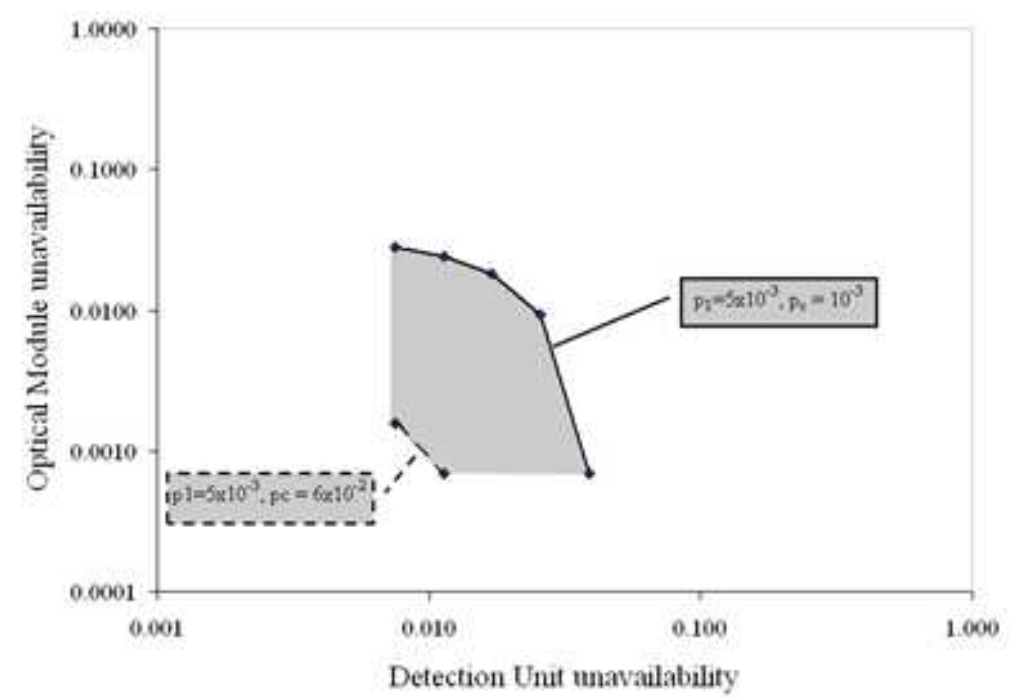

Figure 4: Component unavalabilities for the detector configuration $[17,6,3,20]$

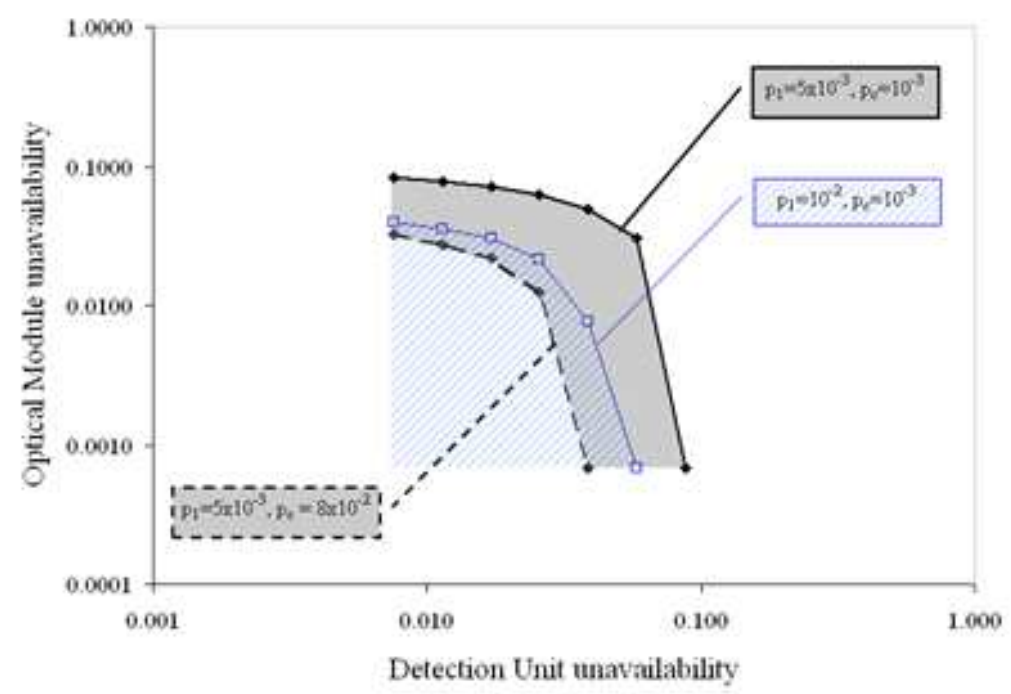

Figure 5: Component unavalabilities for the detector configuration [18, 6, 3, 20]

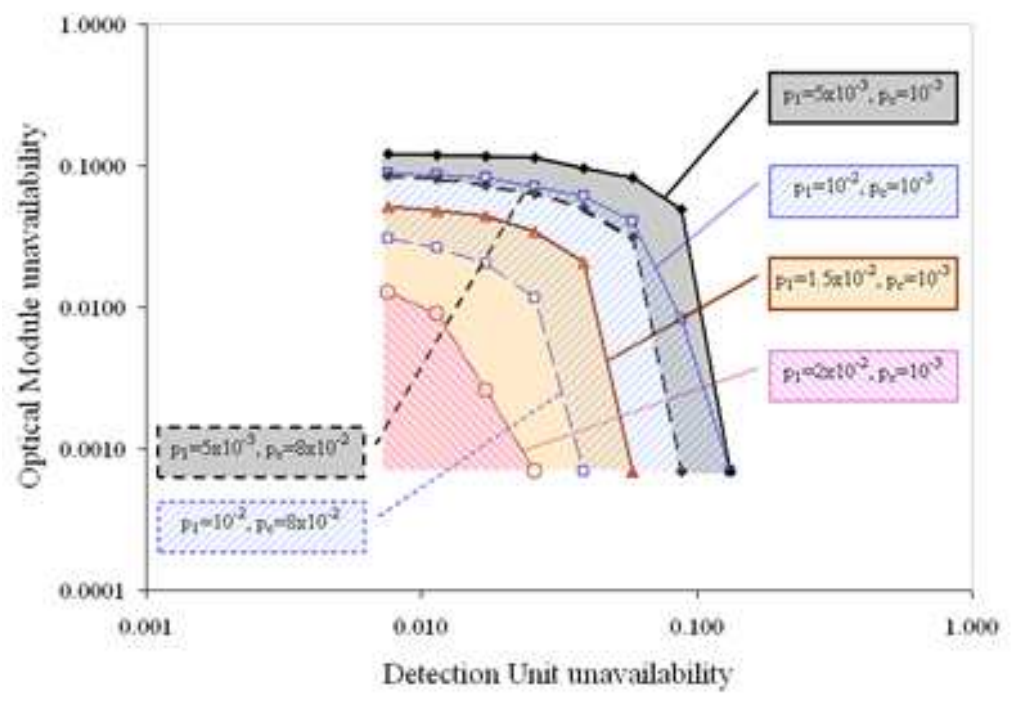

Figure 6: Component unavalabilities for the detector configuration $[19,6,3,20]$ 
$p_{4}=1.6 \times 10^{-3}$, respectively. If $p_{c}=10^{-3}$ the possible combinations of $p_{3}, p_{4}$ unavailabilities are given by the upper limit curve (solid line) in Figure 4. Likewise, for $p_{c}=6 \times 10^{-2}$ the possible $p_{3}, p_{4}$ combinations are given by the lower limit curve (dashed line) in Figure 4 . The gray area indicates the possible values of the triplets $\left(p_{c}, p_{3}, p_{4}\right)$. Higher sector unavailability values violate the constraint on $U_{T}$.

Figure 5 presents the results for the $[18,6,3,20]$ configuration. For this configuration results within the assumed unavailability ranges exist only if the sector unavailability $\left(p_{1}\right)$ is less than $10^{-2}$. If $p_{1}=5 \times 10^{-3}$ the cable unavailability can vary between $8 \times 10^{-2}$ and $10^{-3}$, and the range of possible $\left(p_{3}, p_{4}\right)$ values, for the DU and the OM unavailabilities, is given by the gray area. For $p_{1}=5 \times 10^{-3}$ and $p_{c}=10^{-3}$ the possible combinations of $p_{3}, p_{4}$ unavailabilities are given by the upper limit curve (solid line, bold for $p_{c}=10^{-3}$ ). For $p_{1}=5 \times 10^{-3}$ and $p_{c}=8 \times 10^{-2}$ the possible combinations of $p_{3}, p_{4}$ unavailabilities are given by the lower limit curve (dashed line, bold for $p_{c}=10^{-3}$ ). If $p_{1}$ increases to $10^{-2}$ then lower values for the unavailabilities of the other components are required. The possible values for the cable unavailability are now between $10^{-3}$ and $7 \times 10^{-2}$, while the corresponding $p_{3}, p_{4}$ unavailabilities are contained in the white/blue upward diagonal area in Figure 5.

Figure 6 presents the results for the $[19,6,3,20]$ configuration. For this configuration results within the assumed unavailability ranges exist only for $p_{1}$ less than $2 \times 10^{-2}$. For $p_{1}=5 \times 10^{-3}$ the cable unavailability $p_{c}$ lies between $8 \times 10^{-2}$ and $10^{-3}$ and the range of possible values for $p_{3}$ and $p_{4}$ is given by the gray area. Higher $p_{1}$ values call for lower unavailabilities of the other components. The white/blue upward diagonal area, the orange with bold limiting curves and the white/purple downward diagonal shaded areas correspond to $p_{1}$ values of $10^{-2}$, $1.5 \times 10^{-2}$ and $2 \times 10^{-2}$, respectively.

Figure 7 presents the results for the $[20,6,3,20]$ configuration. For this configuration results within the assumed unavailability ranges exist only for $p_{1}$ less than $2.5 \times 10^{-2}$. For $p_{c}=8 \times 10^{-2}, p_{3}$ drops from $2 \times 10^{-1}$ to $4 \times 10^{-2}$ as $p_{1}$ increases from $5 \times 10^{-3}$ to $2.5 \times 10^{-2}$, respectively. The green area with bold limiting curves corresponds here to $p_{1}$ values of $2.5 \times 10^{-2}$.

Figure 8 presents the results for the $[19,7,3,20]$ configuration. For this configuration results within the assumed unavailability ranges exist only for $p_{1}$ less than $3.5 \times 10^{-2}$. For $p_{c}=8 \times 10^{-2}, p_{3}$ drops from $2 \times 10^{-1}$ at $p_{1}=5 \times 10^{-3}$ to $6 \times 10^{-2}$ at $p_{1}=3.5 \times 10^{-2}$. The black outlined diamond area corresponds to $p_{1}$ values of $3 \times 10^{-2}$. 


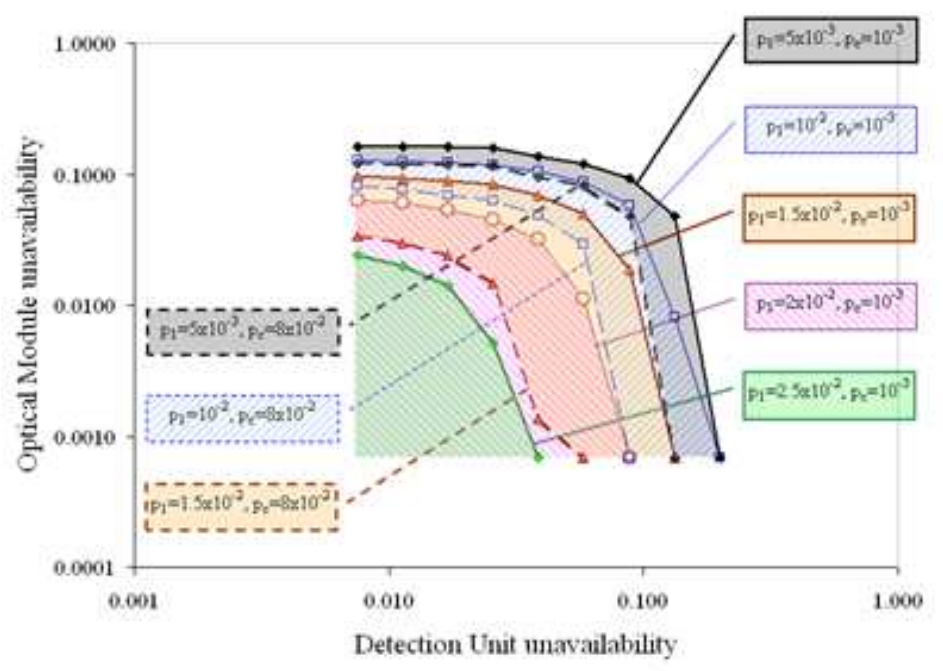

Figure 7: Component unavalabilities for the detector configuration $[20,6,3,20]$

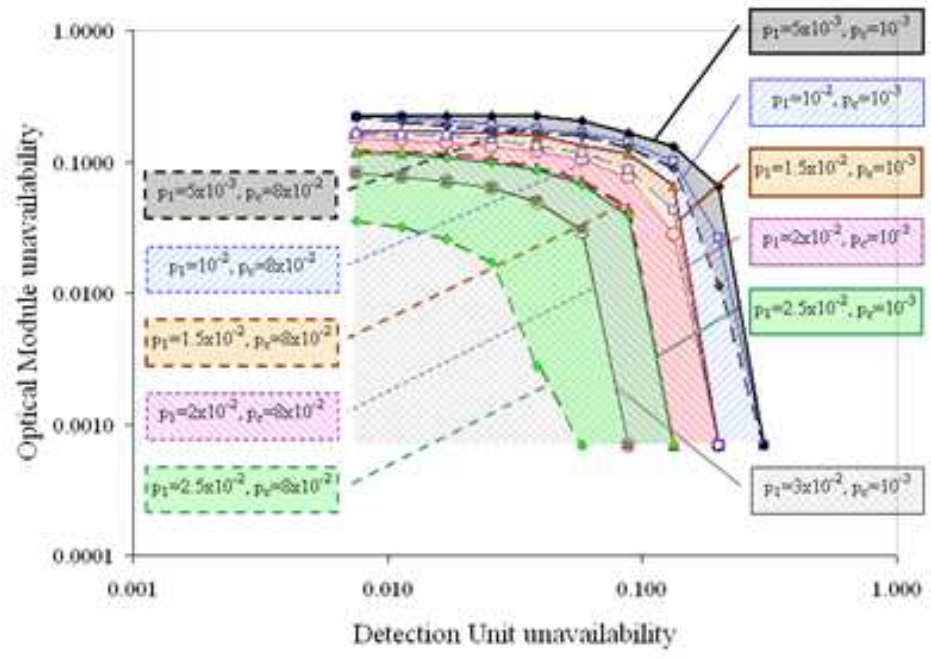

Figure 8 : Component unavalabilities for the detector configuration $[19,7,3,20]$

For the $[20,7,3,20]$ configuration, results within the assumed unavailability ranges exist only for $p_{1}$ less than $4.5 \times 10^{-2}$. For $p_{c}=8 \times 10^{-2}, p_{3}$ is above $2 \times 10^{-1}$ at $p_{1}=5 \times 10^{-3}$ and drops to $1.7 \times 10^{-2}$ as $p_{1}$ increases to $4.5 \times 10^{-2}$. For the $[21,7,3,20]$ configuration, results within the assumed unavailability ranges exist only for $p_{1}$ less than $5 \times 10^{-2}$. For $p_{c}=8 \times 10^{-2}, p_{3}$ is above $3 \times 10^{-1}$ for $p_{1}=5 \times 10^{-3}$ and reduces to $2.5 \times 10^{-2}$ at $p_{1}=5 \times 10^{-2}$.

\subsection{Discussion}

The purpose of this section is to present ranges and combinations of possible component unavailability values that satisfy an overall system unavailability requirement. The unavailability of a component depends on its MTTF as well as on whether the component is repairable, and if yes, how often can the repair be undertaken and how long it lasts. At this phase of the telescope design it is not yet established whether all or which components will be 
retrievable and repairable. For this reason, the present analysis provides an additional way to increase the system unavailability by increasing the number of deployed OMs.

Table 1 reports a representative selection of the results described in section 4.2 and presented in Figures 4 to 8, where

- The main cable unavailability is taken at $6 \times 10^{-2}, 2 \times 10^{-2}, 10^{-2}$ and $10^{-3}$. According to the employed repair policy for the main cable, the respective MTTFs can be 1.3, 4.1, 8.3 and 83 years if immediate repair within one month MTTR is applicable (equation (36)) or 9.2, 28.7, 57.8 and 583 years if a fixed annual repair period of one month is applicable (equation (37)) etc.

- Two sets of values for the unavailabilities of the detector network cable and fibers are considered, namely (a) $p_{1}=5 \times 10^{-3}, p_{2}=2.5 \times 10^{-2}$, and (b) $p_{1}=1.5 \times 10^{-2}, p_{2}=7.5 \times 10^{-2}$. Assuming that all the components in the detector network are non-repairable, equation (35) provides the corresponding MTTFs for the $\left(p_{1}, p_{2}\right)$ sets at 1000, 200 years for (a), and 330, 63 years for set (b).

- Indicatively, the cases discussed here consider that OMs and DUs have the same unavailabilities. Assuming that OMs and DUs are non-repairable, Table 1 provides the MTTFs required to satisfy the system unavailability target $U_{T}=10^{-1}$.

Table 1: Reliability requirements, in the form of MTTFs, for non-repairable OMs and DUs

\begin{tabular}{cccccccc}
\hline main cable & \multicolumn{7}{c}{ detector network configuration \& number of optical modules } \\
unavailability & {$[17,6,3,20]$} & {$[18,6,3,20]$} & {$[19,6,3,20]$} & {$[20,6,3,20]$} & {$[19,7,3,20]$} & {$[20,7,3,20]$} & {$[21,7,3,20]$} \\
$p_{c}$ & 6120 & 6480 & 6840 & 7200 & 7980 & 8400 & 8820 \\
\hline $6 \times 10^{-2}$ & 1160 & 150 & 82.5 & 57.5 & 36.2 & 30.2 & 26.2 \\
$2 \times 10^{-2}$ & 345 & 115 & 70.7 & 51.7 & 33.6 & 28.4 & 24.8 \\
$10^{-2}$ & 305 & 111 & 69.2 & 50.9 & 33.2 & 28.2 & 24.6 \\
$10^{-3}$ & 280 & 108 & 68.0 & 50.3 & 33.0 & 28.0 & 24.2 \\
\hline \hline & (a) minimum MTTF (in years) for $p_{1}=5 \times 10^{-3}, p_{2}=2.5 \times 10^{-3}, p_{3}=p_{4}$ and $U_{T} \leq 10^{-1}$ \\
\hline $6 \times 10^{-2}$ & n.a. & n.a. & 385 & 127 & 55.3 & 42.5 & 34.8 \\
$2 \times 10^{-2}$ & n.a. & 6000 & 185 & 93.5 & 47.7 & 37.9 & 31.7 \\
$10^{-2}$ & n.a. & 2700 & 172 & 90.4 & 46.7 & 37.3 & 31.3 \\
$10^{-3}$ & n.a. & 1900 & 163 & 88.0 & 46.0 & 36.9 & 30.9 \\
\hline
\end{tabular}


In particular

- For $p_{1}=5 \times 10^{-3}, p_{2}=2.5 \times 10^{-2}$ and $p_{c}=6 \times 10^{-2}$, the base configuration $[17,6,3,20]$ requires MTTF of 1160 years for DUs and OMs. If this MTTF is not technically achievable or is extremely expensive then additional redundancy in the form of extra DUs and OMs is required. As the size of the network increases up to $8820 \mathrm{OMs}$, the MTTF requirement for the DUs and the OMs decreases down to 26.2 years. The MTTF requirements decrease also as the unavailability of the main cable decreases down to $10^{-3}$ (see Table 1 (a)).

- The importance of the main cable unavailability is reduced as the network redundancy increases. For example, assuming the base design featuring $6120 \mathrm{OMs}$, as $p_{c}$ drops from $6 \times 10^{-2}$ to $10^{-3}$ the required MTTF for DUs and OMs drops by a factor of 5, from 1160 to 280 years. For the configuration [19, 7, 3, 20] having 7980 OMs, the MTTF for DUs and OMs drops from 36 to 33 years, so the reduction due to the increased availability of the main cable is now only $3 \%$.

- Similar behaviors are observed if the sector and branch unavailabilities increase to $p_{1}=1.5 \times 10^{-2}$ and $p_{2}=7.5 \times 10^{-2}$, respectively (see Table $1(\mathrm{~b})$ ). In this case and for the $p_{c}$ range considered here, the base detector network $[17,6,3,20]$ cannot meet the $U_{T}$ target for any DU and OM MTTF. The $[18,6,3,20]$ network requires a $p_{c} \leq 2 \times 10^{-2}$ and then the required MTTFs for OMs and DUs are in the order of a few thousand years.

These results indicate that the limiting constraint to the telescope unavailability will be set by the technically achievable MTTF and whether some or all of the detector network components may be retrievable and repairable. If repair of these components is not possible then higher numbers of OMs must be deployed and the unavailability model may be used to suggest alternatives to meet the same overall unavailability target.

For example, assume that the manufacturer sets the maximum attainable MTTF for OMs and DUs at 150 years. The smallest network to satisfy the $U_{T}$ target for $p_{1}=1.5 \times 10^{-2}$, $p_{2}=7.5 \times 10^{-2}$ and $p_{c}=6 \times 10^{-2}$ is now the $[20,6,3,20]$, featuring $18 \%$ redundancy compared to the base design. If the manufacturer resets the bound at 100 years, then the options in Table 1 are to apply the configuration $[19,7,3,20]$, or to use $p_{c}=2 \times 10^{-2}$. The latter can also be achieved by manipulating the factors affecting the reliability of the main cable, like distance to shore and routing, deployment and installation etc. Another alternative would be to consider maintenance for the OMs and the DU. The unavailability of a component with 100 
years MTTF and the option to be repaired annually for a fixed period of one month is $5.8 \times 10^{-}$ ${ }^{3}$; same as the unavailability of a non-repairable component with 860 years MTTF. In this case, smaller networks, less expensive to build but more expensive to maintain, like the [19, $6,3,20]$ with $p_{c}=2 \times 10^{-2}$, become a feasible option and the choice depends on the project budget and money flow.

The above discussion demonstrates how the presented model can be used as a decision support tool for determining component reliabilities or adding redundancy to meet a certain overall unavailability requirement. At the end of the day, the optimal telescope configuration is the one that has the minimum total cost of investment, deployment, installation and maintenance during its operation. The choices on alternative network configurations, repair policies, cable routings etc need to consider the relationship between the prescribed component unavailabilities and the cost or the effort for achieving them.

\section{CONCLUSIONS}

This work presents a conceptual dependability analysis for a very large volume submarine neutrino telescope. The dependability criterion used here is quantitative unavailability, and an appropriate unavailability model is developed and presented here. The telescope unavailability is estimated based on the unavailabilities of the telescope components, namely the optical modules, the detection units where the optical modules are hosted, and the cable network transferring the measured neutrino signals to the facilities onshore.

The unavailability model is used to study the effects of different factors on the overall unavailability of the submarine system. Results are presented for different detector network configurations, with detector sizes ranging from 306 detection units of 6120 optical modules up to 441 detection units of 8820 optical modules.

Further research is under way to establish a realistic perspective on the practically attainable component reliability ranges, and develop suitable cost-reliability correlations to depict the trade-offs between component cost and reliability. These correlations will collaborate with the unavailability model developed here to enable a cost-based optimization towards optimal telescope configurations. 


\section{ACKNOWLEDGEMENTS}

This work was partially supported by the EU in FP6, KM3NeT Design Study, Contract Number 011937. The authors would like to thank Dr. Gregory Hallewell for his comments concerning references to the $\mathrm{KM} 3 \mathrm{NeT}$ project.

\section{APPENDIX - COMPONENT STEADY-STATE UNAVAILABILITY}

This section presents an analysis deriving average steady-state unavailabilities for components that are testable and repairable. The derived steady-state unavailabilities will then be used in the relationships of section 3 to calculate the Detection Network unavailability. Note that regardless of the applicable repair regime, the following analysis shows that it is always possible to calculate the average availability of a component over a period of operation.

\section{A.1. System periodically tested with constant repair time}

Consider a system with constant testing period $T$, constant failure rate $\lambda$ and deterministically known and constant repair duration $d$. An often used expression of steady state unavailability of such a system is obtained as follows:

The system unavailability $p_{\mathrm{c}}$ increases from zero as $1-e^{-\lambda t}$, and reaches the value $1-e^{-\lambda T}$ at the instance of the test or

$$
p_{c}(t)=1-e^{-\lambda t} \quad \text { for } \quad 0 \leq t \leq T
$$

During the repair period, $T<t<T+d$, the probability that the system is unavailable, $p_{c}(t)=\operatorname{Pr}[U(t)]$, can be formulated as the joint probability of the "sure event" $\{A(T)+U(T)\}$ that the system is either available or unavailable at time $T$, and the event the system is unavailable at time $t$.

$$
p_{c}(t)=\operatorname{Pr}[U(t)]=\operatorname{Pr}[\{A(T)+U(T)\}, U(t)]
$$

Since the events "system available" $\{A(T)\}$ and "system unavailable" $\{U(T)\}$ are mutually exclusive it follows that

$$
p_{c}(t)=\operatorname{Pr}[A(T), U(t)]+\operatorname{Pr}[U(T), U(t)]
$$

and using conditional probabilities

$$
p_{c}(t)=\operatorname{Pr}[U(t) \mid A(T)] \cdot \operatorname{Pr}[A(T)]+\operatorname{Pr}[U(t) \mid U(T)] \cdot \operatorname{Pr}[U(T)]
$$


since $\quad \operatorname{Pr}[U(t) \mid A(T)]=1-e^{-\lambda(t-T)}, \quad \operatorname{Pr}[A(T)]=e^{-\lambda T}, \quad \operatorname{Pr}[U(t) \mid U(T)]=1 \quad$ and $\operatorname{Pr}[U(T)]=1-e^{-\lambda T}$, this becomes

$$
\begin{aligned}
p_{c}\left(t^{\prime}\right) & =\left(1-e^{-\lambda\left(t^{\prime}-T\right)}\right) \cdot e^{-\lambda T}+1 \cdot\left(1-e^{-\lambda T}\right) \\
& =\left(1-e^{-\lambda\left(t^{\prime}-T\right)}\right) \cdot e^{-\lambda T}+1-e^{-\lambda T} \quad \text { for } T \leq t^{\prime} \leq T+d
\end{aligned}
$$

Setting $x=t^{\prime}-T$ with $0 \leq x \leq d$ and noting that $1-e^{-\lambda x} \cong 1-1+\lambda x-\ldots=\lambda x \leq \lambda d$, if $(\lambda d)$ is very small, that is if potential failures in the short interval of $d$ can be ignored, then the term $\left(1-e^{-\lambda(t-T)}\right) \cdot e^{-\lambda T}$ in the equation above can be ignored and the system availability is given by

$$
p_{c}(t)= \begin{cases}1-e^{-\lambda t} & \text { for } 0 \leq t \leq T \\ 1-e^{-\lambda T} & \text { for } T \leq t \leq T+d\end{cases}
$$

Assuming that the repair is perfect and hence when completed the unavailability drops to zero, the mean unavailability during the interval $0 \leq t \leq T+d$ is given by

$$
\bar{p}_{c}=\frac{1}{T+d} \int_{0}^{T+d} p_{c}(t) d t
$$

yielding

$$
\bar{p}_{c}=\frac{1}{T+d}\left(\frac{\lambda(T+d)+(1-\lambda d) e^{-\lambda T}-1}{\lambda}\right)
$$

If $T$ is such that $\lambda T<<1$ then $e^{-\lambda T} \approx 1-\lambda T+\frac{(\lambda \mathrm{T})^{2}}{2}$ and $\frac{(\lambda \mathrm{T})^{2}}{2}(\lambda d) \approx 0$

Additionally, if $T>>d$ then $\frac{T}{T+d} \approx 1$ and (A.1) reduces to ${ }^{[12-14]}$

$$
\bar{p}_{c}=\frac{1}{2} \lambda T+\lambda d
$$

A more accurate analysis of periodically tested systems with constant time-to-repair is obtained if possible failures during the interval $(T, T+d)$ are taken into account. The statetransition diagram of this system is as shown in Figure 9.

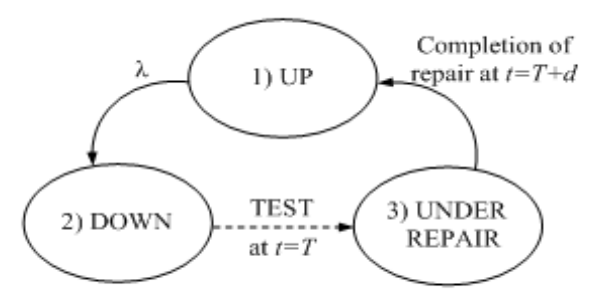

Figure 9: Periodically tested component with constant repair time 
The system exhibits a periodic behavior with period $T^{\prime}=T+d$. Let $\pi_{1}(k), \pi_{2}(k)$, $\pi_{3}(k)$ denote the state probabilities at the end of the $\mathrm{k}^{\text {th }}$ period. It is $\pi_{3}(k)=0$ since, after the completion of the repair-time period $d$, the system, if under repair, transits from state 3 to state 1 with probability equal to unity. The state equations are

$$
\begin{aligned}
& \dot{\pi}_{1}(t)=-\lambda \pi_{1}(t) \\
& \dot{\pi}_{2}(t)=\lambda \pi_{1}(t) \\
& \dot{\pi}_{3}(t)=0
\end{aligned}
$$

Consider the transitions of the system for the next period, i.e. between $k(T+d)$ and $(k+1)(T+d)$, and two intervals in this period: $0<t<T$ and $T<t<T+d$.

In the first interval, $0<t<T$, the state equations (A.3) are solved by applying Laplace transform with initial conditions $\pi_{1}(0)=\pi_{1}(k), \pi_{2}(0)=\pi_{2}(k)$

$$
\begin{aligned}
& \pi_{1}(t)=\pi_{1}(k) e^{-\lambda t} \\
& \pi_{2}(t)=\pi_{2}(k)+\pi_{1}(k)\left(1-e^{-\lambda t}\right) \text { for } 0<t<T \\
& \pi_{3}(t)=\pi_{3}(k)=0
\end{aligned}
$$

For $t=T$, just before the test, equations (A.4) become

$$
\begin{aligned}
& \pi_{1}(T)=\pi_{1}(k) e^{-\lambda T} \\
& \pi_{2}(T)=\pi_{2}(k)+\pi_{1}(k)\left(1-e^{-\lambda T}\right) \quad \mathrm{t}=T \\
& \pi_{3}(T)=\pi_{3}(k)=0
\end{aligned}
$$

For $t=T^{+}$, immediately after the test, equations (A.4) become

$$
\begin{aligned}
& \pi_{1}\left(T^{+}\right)=\pi_{1}(k) e^{-\lambda T} \\
& \pi_{2}\left(T^{+}\right)=0 \\
& \pi_{3}\left(T^{+}\right)=\pi_{2}(k)+\pi_{1}(k)\left(1-e^{-\lambda T}\right)
\end{aligned}
$$

For the second interval, $T<t<T+d$, the state equations (A.3) are solved with

$$
\begin{aligned}
\pi_{1}(0)=\pi_{1}(T), \pi_{2}(0)=0, \pi_{3}(0)=\pi_{3}(T), \text { using } x=t-T \\
\pi_{1}(t)=\pi_{1}(T) e^{-\lambda x} \\
\pi_{2}(t)=\left(1-e^{-\lambda x}\right) \pi_{1}(T) \\
\pi_{3}(t)=\pi_{3}(T)
\end{aligned}
$$

At the end of this period $(x=d)$ these equations become

$$
\begin{aligned}
& \pi_{1}(T+d)=\pi_{1}(T) e^{-\lambda d} \\
& \pi_{2}(T+d)=\left(1-e^{-\lambda d}\right) \pi_{1}(T) \\
& \pi_{3}(T+d)=\pi_{3}(T)
\end{aligned}
$$

Immediately after the completion of the repair $\pi_{3}$ becomes zero and 


$$
\begin{aligned}
& \pi_{1}(T+d)=\pi_{1}(T) e^{-\lambda d}+\pi_{3}(T) \\
& \pi_{2}(T+d)=\pi_{1}(T)\left(1-e^{-\lambda d}\right) \\
& \pi_{3}(T+d)=0
\end{aligned}
$$

By virtue of (A.6) the above relationships give

$$
\begin{aligned}
& \pi_{1}(k+1)=\left[1-e^{-\lambda T}+e^{-\lambda(T+d)}\right] \pi_{1}(k)+\pi_{2}(k) \\
& \pi_{2}(k+1)=\left(e^{-\lambda T}-e^{-\lambda(T+d)}\right) \pi_{1}(k)
\end{aligned}
$$

equations (A.10) provide recursively the state-probabilities $\pi_{1}(k+1), \pi_{2}(k+1)$ after the completion of the $(k+1)^{\text {th }}$ test, in terms of $\pi_{1}(k)$ and $\pi_{2}(k)$. Given the initial conditions $\pi_{1}(0)=1, \pi_{2}(0)=0$, the equations can be solved for any value of $k$ as

$$
\begin{aligned}
& \pi_{1}(k)=\frac{b}{\alpha+b}+(1-\alpha-b)^{k} \frac{\alpha}{\alpha+b} \\
& \pi_{2}(k)=\frac{\alpha}{\alpha+b}-(1-\alpha-b)^{k} \frac{\alpha}{\alpha+b}
\end{aligned}
$$

where $\alpha=e^{-\lambda T}\left(1-e^{-\lambda d}\right)$ and $b=1$.

The unavailability of the system is the probability of occupying state 2 after the completion of the repair at the end of the $k^{\text {th }}$ period of $(T+d)$, is therefore

$$
p_{c}(k)=\frac{\alpha}{\alpha+b}-(1-\alpha-b)^{k} \frac{\alpha}{\alpha+b}
$$

Since $\lim _{k \rightarrow \infty}(1-a-b)^{k}=\lim _{k \rightarrow \infty}\left(e^{-\lambda T}\left(1-e^{-\lambda d}\right)\right)^{k}=0$, equation (A.12) converges to an asymptotic steady-state solution. Therefore,

$$
p_{c}(\infty)=\frac{e^{-\lambda T}-e^{-\lambda(T+d)}}{1+e^{-\lambda T}-e^{-\lambda(T+d)}} \text { and } A(\infty)=1-p_{c}(\infty)=\frac{1}{1+e^{-\lambda T}-e^{-\lambda(T+d)}}
$$

Note that, for $\lambda(T+d)<<1, e^{-\lambda T} \approx 1-\lambda T$ and $e^{-\lambda(T+d)} \approx 1-\lambda(T+d),($ A.13) reduces to

$$
p_{c}(\infty)=\frac{\lambda d}{1+\lambda d}=\frac{d}{\frac{1}{\lambda}+d}
$$

which is the steady-state unavailability for all types of failure and repair ${ }^{[11]}$.

Between successive periods (from $t=0$ until $t=T+d$ ) even when the steady-state has been achieved the unavailability changes with time, according to (A.4) for $0<t<T$ and (A.7) for $T<t<T+d$.

The average unavailability is defined as 


$$
\bar{p}_{c, \text { int }}(k, k+1) \equiv \frac{1}{T+d} \int_{0}^{T+d} p_{c}(t) d t=\frac{1}{T+d}\left[\int_{0}^{T} p_{c}(t) d t+\int_{T}^{T+d} p_{c}(t) d t\right]
$$

equation (A.4) gives

$$
\int_{0}^{T} p_{c}(t) d t=\pi_{2}(k) T+\pi_{1}(k)\left[T-\frac{1-e^{-\lambda T}}{\lambda}\right]=T-\pi_{1}(k) \frac{1-e^{-\lambda T}}{\lambda}
$$

while equation (A.7) gives

$$
\int_{T}^{T+d} p_{c}(t) d t=d-\pi_{1}(k) \frac{e^{-\lambda T}-e^{-\lambda(T+d)}}{\lambda}
$$

Using (A.16) and (A.17), equation (A.15) becomes

$$
\bar{p}_{c, \text { int }}(k, k+1)=1-\pi_{1}(k) \frac{1-e^{-\lambda(T+d)}}{\lambda(T+d)}
$$

When the steady state is achieved, then $\pi_{1}(k)=\pi_{1}(\infty)=1-p_{c}(\infty)$ and by virtue of (A.13) it follows that

$$
\bar{p}_{c, \text { int }}(\infty)=1-\frac{1-e^{-\lambda(T+d)}}{\lambda(T+d)\left(1+e^{-\lambda T}-e^{-\lambda(T+d)}\right)}
$$

Note that, for $\lambda(T+d)<<1$ equation (A.19) is reduced to

$$
\bar{p}_{c, \text { int }}(k, k+1)=1-\pi_{1}(k) \frac{\lambda(T+d)}{\lambda(T+d)}=1-\pi_{1}(k)=\pi_{2}(k)
$$

This means that time dependence of the unavailability within the period is not important and $\bar{p}_{c, \text { int }}(k, k+1)$ is equal to the unavailability at the end of the $k^{\text {th }}$ period $\pi_{2}(k)$.

An interesting special case is when the repair (of constant duration) can start immediately after the failure. This is equivalent to having $T \rightarrow 0$ and equation (A.19) yields

$$
\bar{p}_{c, \text { int }}(\infty)=1-\frac{1-e^{-\lambda d}}{\lambda d\left(2-e^{-\lambda d}\right)}
$$

and of course for $\lambda d \ll 1$ this reduces to

$$
\bar{p}_{c, \text { int }}(\infty)=\frac{d}{\frac{1}{\lambda}+d}=\frac{d}{M T T F+d}
$$

This relationship is valid for any type of probability distribution functions for the times to failure and times to repair ${ }^{[11]}$. 


\section{REFERENCES}

1. Becker JK. High-energy neutrinos in the context of multimessenger astrophysics. Phys Rep 2008; 458(4-5):173-246. doi:10.1016/j.physrep.2007.10.006

2. Berghaus $P$, the IceCube Collaboration. IceCube: Status and first results. Nucl Phys B (Proc Suppl) 2009; 190:127-32. doi:10.1016/j.nuclphysbps.2009.03.078

3. Distefano C, the KM3NeT Consortium. KM3NeT: Towards a $\mathrm{km}^{3}$-scale neutrino telescope in the Mediterranean Sea. Nucl Phys B (Proc Suppl) 2009; 190:115-20. doi:10.1016/j.nuclphysbps.2009.03.076

4. Katz UF. Underwater neutrino telescopes. Nucl Phys B (Proc Suppl) 2009; 188:24551. doi:10.1016/j.nuclphysbps.2009.02.057

5. Katz UF, for the KM3NeT Consortium. Status of the KM3NeT project. Nucl Instrum Methods Phys Res A 2009; 602(1):40-6. doi:10.1016/j.nima.2008.12.215

6. Abbasi R, Ackermann M, Adams J, Ahlers M, Ahrens J, K. Andeen, J, et al. The IceCube data acquisition system: Signal capture, digitization, and timestamping. Nucl Instrum Methods Phys Res A 2009; 601(3):294-316. doi:10.1016/j.nima.2009.01.001

7. Kooijaman P, Carr J, Hallewell G, Holford A, Katz U, Moscoso L, Piattelli P, Rapidis P, editors. Conceptual design report for a deep-sea research infrastructure incorporating a very large volume neutrino telescope in the Mediterranean sea. 2008 April [cited 2010 May 06]. Available from: http://www.km3net.org/CDR/CDRKM3NeT.pdf

8. Tsirigotis AG, Tzamarias SE. Application of Kalman filter methods to event filtering and reconstruction for neutrino telescopy. Nucl Instrum Methods Phys Res A 2009; 602(1):91-4. doi:10.1016/j.nima.2008.12.005

9. Davenport WB. Probability and random processes: An introduction for applied scientists and engineers. New York: McGraw-Hill; 1970.

10. Rausand M, Hoyland A. System Reliability Theory - Models, Statistical Methods and Applications, $2^{\text {nd }}$ Edition, Chapter 10, Wiley, 2004. 
11. Kumamoto H, Henley EJ. Probabilistic risk assessment and management for engineers and scientists. Chapter 6, New York: IEEE Press, 1996.

12. Zio E. An Introduction to the basics of Reliability and Risk Analysis, Section 6.6.1, World Scientific Publishing Co. Pte. Ltd., Singapore, 2007.

13. Fault Tree Handbook with Aerospace Applications, NASA Office of Safety and Mission Assurance, NASA, Washington Dc, USA, June 2002.

14. Procedures for Conducting Probabilistic Safety Assessments of Nuclear Power Plants (Level1), Table XI, items 1.1 and 1.3, Safety Series No. 50-P-4, IAEA Vienna, 1992.

\section{TABLE CAPTIONS}

Table 1: Reliability requirements, in the form of MTTFs, for non-repairable OMs and DUs

\section{FIGURE CAPTIONS}

Figure 1: Overall neutrino telescope system

Figure 2: Three-dimensional detector network layout

Figure 3: Detector Network and Deep Sea Infrastructure Block Diagrams

Figure 4: Component unavailabilities for the detector configuration $[17,6,3,20]$

Figure 5: Component unavailabilities for the detector configuration $[18,6,3,20]$

Figure 6: Component unavailabilities for the detector configuration $[19,6,3,20]$

Figure 7: Component unavailabilities for the detector configuration $[20,6,3,20]$

Figure 8: Component unavailabilities for the detector configuration $[19,7,3,20]$

Figure 9: Periodically tested component with constant repair time 